\title{
IMPACT of distance learning on student mental health in the COVID-19 pandemic
}

\author{
Nikolay Lyubetsky*, Nikita Bendersky, Tatyana Verina, Lyudmila Demyanova, and Darya Arkhipova
}

Don State Technical University, 344022, Rostov-on-Don, Russia

\begin{abstract}
Education is one of the industries significantly affected by the novel coronavirus infection (COVID-19) pandemic. Whole countries and cities were isolated, public and private institutions ceased their activities, and the country's higher educational institutions were forced to stop fulltime education and switch to distance learning. Thus, the purpose of this study was to compare the indicators of the mental state (depression, anxiety) of students during full-time and distance learning. The first study was carried out by us during the full-time study period (February 10-17, 2020 , sample size $\mathrm{n}=154$ ), and the second study was conducted during the distance learning period (October 19-26, 2020, sample size $n=154$ ). The study evaluated such indicators as satisfaction with academic performance, the severity of depressive symptoms and anxiety. The results obtained indicate that the prevalence of depressive symptoms and anxiety among students was significantly higher during the distance learning period compared to similar indicators obtained during the full-time study period. The results of this study indicate that a sudden shift from one learning regime to another was a causative factor in stressful stress, which led to a high prevalence of depressive symptoms and anxiety among undergraduate students.
\end{abstract}

\section{Introduction}

The turn of 2019-2020 forever entered the history of mankind as the era of the pandemic of the new coronavirus infection, which showed the fragility of our civilization with all its apparent perfection and technological progress. The COVID-19 pandemic has exposed the shortcomings of national health systems. As practice has shown, many countries were unable to implement operational monitoring of the situation, to provide sufficient personal protective equipment for medical workers and the population as a whole [1]. According to analysts, the coronavirus has dealt a serious blow to the global economy. The work of a huge number of industrial enterprises was suspended. Small and medium-sized businesses have been seriously affected [2].

The unprecedented measures introduced by the governments of different countries to prevent the spread of the new coronavirus infection, including various restrictions and prohibitions, have minimized direct social contact.

\footnotetext{
${ }^{*}$ Corresponding author: lyudmila-d2@yandex.ru
} 
One of the industries significantly affected by the new coronavirus infection (COVID19) pandemic is education [3]. Whole countries and cities were isolated, public and private institutions ceased their activities, and higher education institutions were forced to stop fulltime education. Some universities had to extend their holidays or end the semester ahead of schedule in order to take measures to prevent infection, postpone the return of students to campus and buy time to decide on the possibility of continuing the quality educational process. Faced with the risk of missing an entire semester or even more, an academic year, universities were forced to integrate distance learning elements into their educational programs to ensure the continuous educational process of students [4].

Over the past 20 years, many experiments have been carried out regarding the application of online learning in higher education institutions, where various courses and even complete undergraduate and graduate programs have been provided through online platforms. However, students studying full-time before this and not familiar with this training format faced many difficulties [5].

The forced transition to a distance learning format has become a new challenge for students. Given the fact that students in "normal conditions" (in full-time mode of study) are sometimes prone to the development of mental disorders, in a pandemic accompanied by social distancing, increased media attention to this problem and lack of information about the new coronavirus infection, only aggravated the situation [6, 7, 8]. Majumdar P. and other authors note that chronic stress caused by the pandemic has led to more frequent manifestations among students of symptoms such as headache, insomnia, digestive problems, hormonal imbalance, frustration, fatigue, etc. [9]. Also, students showed a decrease in cognitive engagement, attention, concentration and motivation to attend classes and study in general $[10,11]$. This information is confirmed by the work of Ahmad S.H. and other authors, which examined the psychosomatic impact of digital tools used in the context of the new coronavirus pandemic on the well-being of students. As a result of the analysis it was revealed that more than $55 \%$ of respondents during the COVID-19 pandemic had changes in their usual sleep patterns, and more than $80 \%$ of students said that prolonged use of digital tools (mobile phone, laptop, iPad) for learning influenced their sleep patterns, and more than $85 \%$ of respondents agreed with the statement that prolonged use of e-learning tools often leads to nervousness and tension. As a result, more than $72 \%$ of students stated that they do not recommend continuing to use the distance learning model, since it is harmful to health in social and psychological terms [12]. Summarizing all of the above, it can be noted that now the entire world community is observing only the primary / short-term consequences associated with the pandemic of a new coronavirus infection, however, the long-term consequences have yet to be clarified [13].

Thus, the purpose of this study is to compare the indicators of the mental state (depression, anxiety) of medical students during full-time and distance learning.

\section{Methods for collecting information and researching psychological factors of the influence of different forms of education on students}

An electronic questionnaire was used for collecting information. Students answered the proposed questions anonymously. The variables in this case were gender, training form, satisfaction with academic performance, the level of depression and anxiety of students.

The study involved 3rd year students of Rostov State Medical University. Participants were asked to fill out an online questionnaire created on the GoogleForms platform. Before testing, each respondent was provided with a voluntary informed consent form, which provided information about the purpose of the study, confidentiality of information and the 
right to withdraw from participation without prior justification. The study was carried out in several stages:

- The first part of the study was conducted during the full-time study (OE) period (February 10-17, 2020)

- The second part of the study was carried out during the period of distance learning (DL) (October 19-26, 2020)

The total number of respondents was 154 people, the average age was 22.3 years (ranging from 21 to 29 years). In the second sample, after switching to the distance learning format, 154 people also took part. The total number of males in both studies was 54 (35.06\%), and $100(64.94 \%)$ were females.

The Likert questionnaire was developed and introduced in Russian, because it is the official language in the Russian Federation. It included several main sections:

1) Socio-demographic and personal characteristics (gender, age, etc.).

2) Questionnaires for assessing psychopathological disorders and the severity of symptoms.

The method of psychometric scaling was used for the purpose of a formalized statistical assessment of psychopathological disorders and the severity of symptoms in medical students. The psychometric study contained the results of objective scales, namely: PHQ-9 - express scale for assessing depression, GAD-7 - express scale for assessing the level of anxiety.

To assess the level of depression, the Patient Health Questionnaire (PHQ-9) was used [14]. This easy-to-use patient questionnaire is a stand-alone version of the PRIME-MD diagnostic tool for general mental disorders. PHQ-9 is a depression module that scores each of the nine criteria of the DSM-IV (Diagnostic and Static Guide to Mental Disorders) as "0 points" (no) to "3 points" (almost every day). The overall score was calculated and interpreted as follows: 0-4 points (minimal depression), 5-9 points (mild depression), 10-14 points (moderate depression), 15-19 points (severe depression) and 20-27 (extremely severe depression). Respondents with a score of more than 10 were considered depressive [15].

Test for the presence of generalized anxiety disorder was used for assesing the level of anxiety, the GAD-7 [16]. The scale is a test of 7 questions, each of which offers four answers. The total score was calculated and interpreted as follows: 0-4 points (minimum level of anxiety), 5-9 points (moderate level of anxiety), 10-14 points (medium level of anxiety), and 15-21 points (high level of anxiety). Research results indicate that this questionnaire has good sensitivity and specificity when the number of points scored is not less than 10 . Respondents with a total of more than 10 points were considered alarming [15].

All procedures performed in our work were in accordance with the ethical standards of the institutional and / or National Research Committee, as well as the 1964 Declaration of Helsinki and its later amendments or comparable ethical standards. Informed consent was obtained from all individual participants involved in the data collection process.

\section{Results of statistical processing of experimental data}

Statistical analysis was carried out using Microsoft Excel and the SPSS statistical software package. The difference between the compared samples was considered significant at a significance level of $\mathrm{p}<0.05$.

Descriptive statistics were performed with the calculation of the mean (M) and standard deviation (SD). To assess the significance of differences between the compared samples between the variables, the Student's t-test was used. To compare the results within the same sample, the ANOVA analysis with the post hoc Bonferroni test was used. A logistic 
regression analysis was performed for assessing the associations of the independent variables,

1. Socio-demographic data

The sample consisted of 154 students, of which 54 (35.06\%) were boys and 100 (64.94\%) were girls. The age of the respondents ranged from 21 to 29 years old, with the average age being 21.2 years $( \pm 1.43)$ (Table 1$)$.

Table 1. Socio-demographic data

\begin{tabular}{|c|c|c|c|}
\hline & & $\mathbf{n}$ & $\mathbf{( \% )}$ \\
\hline \multirow{2}{*}{ Gender } & Male & 54 & $35.06 \%$ \\
\cline { 2 - 4 } & Female & 100 & $64.94 \%$ \\
\hline \multirow{2}{*}{ Age } & Min $/$ & Average & SD \\
\cline { 2 - 4 } & $21-29$ лет & 21.2 & 1.43 \\
\hline
\end{tabular}

2. Satisfaction with academic performance during the period of full-time and online education

We conducted a descriptive analysis to assess the satisfaction of medical students with their academic performance during face-to-face and online education during the COVID-19 pandemic. The results showed that more than a third of the sample, namely 59 (38.31\%) students, remained dissatisfied with their academic performance after switching to distance learning. About 25.97\% $(\mathrm{n}=40)$ of students were completely dissatisfied, $22.08 \%(\mathrm{n}=34)$ were neutral about this, and only $13.64 \%(n=21)$ of respondents were satisfied with their academic performance (Table 2).

Table 2. Satisfaction with academic performance

\begin{tabular}{|l|c|c|c|c|}
\hline \multirow{2}{*}{} & \multicolumn{2}{|c|}{ Face-to-face training } & \multicolumn{2}{c|}{ Distance learning } \\
\cline { 2 - 5 } & $\boldsymbol{n}$ & $\mathbf{( \% )}$ & $\mathbf{n}$ & $\mathbf{( \% )}$ \\
\hline Very dissatisfied & 8 & $5.19 \%$ & 40 & $25.97 \%$ \\
\hline Dissatisfied & 18 & $11.69 \%$ & 59 & $38.31 \%$ \\
\hline Neutral & 32 & $20.78 \%$ & 34 & $22.08 \%$ \\
\hline Satisfied & 96 & $62.34 \%$ & 21 & $13.64 \%$ \\
\hline
\end{tabular}

3. Assessment of the level of depression and anxiety

A further descriptive analysis was performed to assess the prevalence of depressive symptoms and anxiety among medical students before and during the COVID-19 pandemic. The results of the study showed that during the period of full-time study (initial level), the average score of students on the PHQ-9 depression scale was 7.63, and on the GAD-7 anxiety scale - 7.24. During the period of distance learning, the average indicator on the PHQ-9 depression scale was 10.85, and on the GAD-7 anxiety scale -9.67 . The increase in the indicators of depression and anxiety for this period was $41.46 \%$ and $33.37 \%$, respectively (Table 3).

Table 3. Assessment of the level of depression and anxiety according to the PHQ-9 and GAD-7 scales.

\begin{tabular}{|c|c|c|c|c|c|c|}
\hline Form of study & Indicators & Min & Max & $\begin{array}{c}\text { Average } \\
\text { value }\end{array}$ & Increase & SD \\
\hline Full-time education & Depression & 0 & 26 & 7.63 & & 5.58 \\
\cline { 2 - 7 } & Anxiety & 0 & 19 & 7.24 & & 4.74 \\
\hline \multirow{2}{*}{$\begin{array}{c}\text { Distance Learning } \\
\text { Depression }\end{array}$} & $\begin{array}{c}\text { Depression } \\
\text { (PHQ-9) }\end{array}$ & 0 & 27 & 10.85 & $\begin{array}{c}+3.18 \\
(41.46 \%)\end{array}$ & 5.90 \\
\cline { 2 - 7 } & $\begin{array}{c}\text { Anxiety } \\
\text { (GAD-7) }\end{array}$ & 0 & 21 & 9.67 & $\begin{array}{c}+2.42 \\
(33.37 \%)\end{array}$ & 5.03 \\
\hline
\end{tabular}


Note: $(*)$ - the value in parentheses shows the increase in $\%$ compared to the baseline.

Legend: SD - standard deviation

Further, in accordance with the threshold values, we analyzed the results shown by students during the questionnaire, where the level of depression and anxiety was assessed (Table 4). The results of the analysis showed that during the full-time study, depression was detected in $29.87 \%(n=46)$ respondents. During the period of distance learning, depressive symptoms were observed in $51.3 \%$ of respondents $(\mathrm{p}<0.001)$.

Table 4. Prevalence of depression among students during full-time and distance learning.

\begin{tabular}{|l|c|c|c|c|}
\hline \multirow{2}{*}{} & \multicolumn{2}{|c|}{ Face-to-face training } & \multicolumn{2}{c|}{ Distance learning } \\
\cline { 2 - 5 } & \multicolumn{2}{|c|}{ Depression } & \multicolumn{2}{c|}{ Depression } \\
\cline { 2 - 5 } & $\mathrm{n}$ & $(\%)$ & $\mathrm{n}$ & $(\%)$ \\
\hline Minimal depression (MD) & 83 & $53.9 \%$ & 54 & $35.06 \%$ \\
\hline Mild depression(LD) & 26 & $16.88 \%$ & 21 & $13.64 \%$ \\
\hline Moderate depression (MD) & 22 & $14.29 \%$ & 42 & $27.27 \%$ \\
\hline Severe depression (SD) & 15 & $9.74 \%$ & 17 & $11.04 \%$ \\
\hline $\begin{array}{l}\text { Extremely severe depression } \\
\text { (ESD) }\end{array}$ & 9 & $5.84 \%$ & 20 & $12.99 \%$ \\
\hline
\end{tabular}

Note: Respondents with a score of more than 10 were considered depressive [15]. Thus, the total number of students with depressive symptoms was calculated as follows - UD + TD + KTD

During the period of full-time education, anxiety was noted in $24.03 \%$ of students, and during distance learning - in $42.86 \%$ ( $\mathrm{p}<0.001$ ) (Table 5). Regardless of gender, the prevalence of anxiety was significantly lower among full-time students $(p<0.05)$. During the period of distance learning, anxiety states were significantly more frequent in women $(\mathrm{p}$ $<0.05)$.

Table 5. Prevalence of anxiety, among students in the period of full-time and distance learning

\begin{tabular}{|l|c|c|c|c|}
\hline \multirow{2}{*}{} & \multicolumn{2}{|c|}{ Face-to-face training } & \multicolumn{2}{c|}{ Distance learning } \\
\cline { 2 - 5 } & \multicolumn{2}{|c|}{ Anxiety } & $\mathrm{n}$ & Anxiety \\
\cline { 2 - 5 } & $\mathrm{n}$ & $(\%)$ & 45 & $29.22 \%$ \\
\hline Minimum (MT) & 79 & $51.3 \%$ & 43 & $27.92 \%$ \\
\hline Moderate (UT) & 38 & $24.68 \%$ & 37 & $24.03 \%$ \\
\hline Medium (ST) & 31 & $20.13 \%$ & 29 & $18.83 \%$ \\
\hline High (HT) & 6 & $3.9 \%$ & \multicolumn{2}{c}{ (HT) } \\
\hline
\end{tabular}

Note: Respondents with a score of more than 10 were considered alarming [15]. Thus, the total number of students with identified anxiety was calculated as follows - ST + VT

4. The difference in student satisfaction with their academic performance, depending on the form of education.

Subsequently, we conducted an independent t-test to determine the difference in student satisfaction with their academic performance during distance learning, taking into account gender differences. The results of our study did not show significant differences in satisfaction with academic performance depending on gender, however, there was a significant difference between the groups of full-time and distance learning $(p=0.00)$ (Table 6).

Table 6. Differences in student satisfaction with academic performance during distance learning.

\begin{tabular}{|l|c|c|c|c|c|}
\hline \multicolumn{2}{|c|}{} & t & M & SD & p \\
\hline \multirow{2}{*}{ Satisfaction with academic performance } & Male & -1.11 & 2.48 & 1.12 & \multirow{2}{*}{0.69} \\
\cline { 3 - 6 } & Female & -1.19 & 2.53 & 1.21 & \\
\hline \multicolumn{2}{|c|}{} & df & M & F & p \\
\hline Satisfaction with academic performance & Between groups & 13 & 4,01 & 3,99 & 0.00 \\
\hline
\end{tabular}


Note: Values in bold indicate statistical significance $\mathrm{p}<0.05$.

Legend: $S D$ - standard deviation, $p$ - significance level, $d f$ - degree of freedom, $F$ - regression significance, $t$ - t-criterion for the free term, $M$ - mean value.

5. The relationship between satisfaction with the academic performance of medical students, the scale of depression and anxiety.

In the course of the study, a two-dimensional correlation analysis was carried out to determine the relationship between satisfaction with student progress during distance learning and the scale of depression and anxiety (Table 7). The analysis results showed a significant negative correlation between the indicated variables $(\mathrm{p}=0.00)$. This indicates that the severity of depressive symptoms and anxiety during the period of distance learning had a significant negative impact on student satisfaction with their academic performance.

Table 7. Relationship Between Student Satisfaction, Depression, and Anxiety

\begin{tabular}{|c|c|c|c|}
\hline & & Depression & Anxiety \\
\hline \multirow{2}{*}{ Academic satisfaction } & R-value & -0.41 & -0.32 \\
\cline { 2 - 4 } & P-value & 0.00 & 0.00 \\
\hline
\end{tabular}

Note: Values in bold indicate statistical significance $\mathrm{p}<0.05$.

Legend: $P$ - value - significance level, $R$ - value - correlation coefficient.

6. Satisfaction with academic performance as a predictor of the development of depressive symptoms and anxiety.

A regression analysis was carried out in order to answer the questions posed to us. The results of analysis showed that satisfaction with academic performance is an important predictor (prognostic parameter) for determining the prevalence of both depressive symptoms $(\mathrm{p}=0.00)$ and anxiety $(\mathrm{p}=0,00)($ Table 8$)$.

Table 8. Satisfaction with academic performance as a predictor of the development of depressive symptoms and anxiety.

\begin{tabular}{|l|c|c|c|c|c|}
\hline & B & SE & Beta & T & P-value \\
\hline Depression & 11,23 & 0.59 & -0.41 & 17,45 & $\mathbf{0 . 0 0}$ \\
\hline Anxiety & 10.29 & 0.53 & -0.032 & 18.83 & $\mathbf{0 . 0 0}$ \\
\hline
\end{tabular}

Note: Values in bold indicate statistical significance $\mathrm{p}<0.05$.

Legend: $p$-value - level of significance, $t$ - t-test for the free term of the equation, $S E$ - standard error, $B$ - regression coefficient, Beta - coefficients of the equation

\section{Discussion}

The research took place during 2020 academic year (February-October). The participantswere 3rd year students of the Rostov Medical University. The researchrealized the fact that a change in the learning regime (transition from full-time to distance learning) is one of the negative factors affecting the mental health of students. The results showed that satisfaction with academic performance among students was significantly higher during the period of full-time education - $62.34 \%$, versus $13.64 \%$ - distance learning. These results are consistent with the work of Olivet J. It was found that higher satisfaction with academic performance is observed with the traditional form of education [17].

We found that the prevalence of depressive symptoms was $29.97 \%$ - full-time education, versus $51.3 \%$ - distance learning and anxiety $24.03 \%$ - full-time education, versus $42.86 \%$ - distance learning increased significantly after students switched to a 
distance format learning. We believe that one of the serious factors that influenced the mental health of students in the context of distance learning is the lack of socialization, communication with classmates and teachers, that is, social loneliness. A number of studies indicate that students interacting with their colleagues show greater involvement, motivation, and also demonstrate higher academic performance [18, 19]. However, the restrictive measures introduced to prevent the spread of the new coronavirus infection minimized direct social contact, which, in our opinion, influenced the mental state of students, and also caused a high prevalence of anxiety and depression among medical students.

Also, in our study, it was found that student satisfaction with their academic performance is an important predictor of the development of depression and anxiety among students of a medical university $(\mathrm{p}=0.00)$.

Thus, in the event of a repetition of such a situation requiring the introduction of quarantine measures and the transition to distance learning, it is necessary to develop and introduce new mechanisms at the Higher School to increase interaction between students.

\section{Conclusions}

The forced transition to a distance learning format has become a new challenge for students. This study, conducted by us among students of the Rostov Medical University, revealed significant changes in the mental state of students, which were manifested by depressive symptoms and anxiety. We found that the prevalence of depressive symptoms $(29.97 \%$ versus $51.3 \%)$ and anxiety $(24.03 \%$ versus $42.86 \%)$ among students was significantly higher during the distance learning period compared to similar indicators obtained during the period full-time training. Satisfaction with academic performance was also significantly higher during the period of full-time study compared with the period of distance learning (62.34\% versus $13.64 \%)$. We found a significant relationship between satisfaction with academic performance, depression and anxiety $(\mathrm{p}=0.00)$, which indicates that the severity of depressive symptoms and anxiety during distance learning had a significant negative impact on student satisfaction with their academic performance. Summarizing all of the above, we can conclude that the sudden transition from full-time education to distance learning was the factor that exerted a stressful load on medical university students, which became the reason for the high prevalence of depressive symptoms and anxiety. Based on the results of our research and on the basis of our professional experience, we believe that the full-time education format (compared to distance education) is more preferable in terms of acquiring high-quality knowledge, contributes to the upbringing and socialization of a physically and mentally healthy person, which is a necessary condition for the self-preservation of society and the state.

\section{References}

1. O.M. Drapkina, I.V. Samorodskaya, M.G. Sivtseva, E.P. Kakorina, N.I. Briko, S.N. Cherkasov, V.A. Tsinzerling, P.G. Malkov, Cardiovascular therapy and prevention 19 (3), 2585 (2020). doi: 10.15829 / 1728-8800-2020-2585

2. H.M. Bai, et al., Int. J. Surg 8 (4), 8-17 (2020).

3. K. Chaturvedi, D.K. Vishwakarma, N. Singh, Children and youth services review 121, 105866 (2021).

4. W. Cao, Z. Fang, G. Hou, M. Han, X. Xu, J. Dong, \& J. Zheng, Psychiatry research 287, 112934 (2020). doi.org/10.1016/j.psychres.2020.112934 
5. A. Ngampornchai, J. Adams, Int J Educ Technol High Educ 13, 34 (2016). doi.org/10.1186/s41239-016-0034-X

6. N. Othman, F. Ahmad, El Morr C., P. Ritvo, International journal of mental health systems 13 (1), 1-9 (2019).

7. N. Vindegaard, M.E. Benros, Brain BehavImmun S0889-1591 (20) 30954-5 (2020).

8. S. Griggs, Journal of psychosocial nursing and mental health services 55 (2), 28-35 (2017)..

9. P. Majumdar, A. Biswas, S. Sahu, Chronobiology International 37 (8), 1191-1200 (2020).

10. Z. Duraku, L. Hoxha, A study of inter action among. The Lancet 395, 10227 (2020).

11. A. Patricia, Int J Educ Res Open, 100011 (2020). doi.org/10.1016/j.ijedro.2020.100011.

12. A.S. Haider, S. Al-Salman, Data Brief, 106104 (2020). doi.org/10.1016/j.dib.2020.106104

13. E. Mahase, Paper advises BMJ 2020 369, 1515 (2020). doi: 10.1136 / bmj.m1515

14. K. Kroenke, R.L. Spitzer, Psychiatr Ann 32 (9), 509-15 (2002). doi.org/10.3928/00485713-20020901-06

15. K. Kroenke, R.L. Spitzer, J.B. Williams, B. Löwe, Gen Hosp Psychiatry 32 (4), 345 59 (2010). doi.org/10.1016/j.genhosppsych.2010.03.006.

16. R.L. Spitzer, K. Kroenke, J.B. Williams, B. Löwe, Arch Intern Med. 166 (10), 1092-7 (2006). doi.org/10.1001/archinte.166.10.1092.

17. J. Olivet, S. Zerger, R.N. Greene, R.R. Kenney, D.B. Herman, Am J Dist Educ. 30, 237-49 (2016).

18. C. Furrer, E. Skinner, J Educ Psychol. 95 (1), 148-62 (2003). doi.org/10.1037/00220663.95.1.148.

19. G.A. Liem, A.J. Martin, British J Educ Psychol. 81 (2), 183-206 (2011). doi.org/10.1111/j.2044-8279.2010.02013.x. 